\title{
Identification of RECK as an evolutionarily conserved tumor suppressor gene for zebrafish malignant peripheral nerve sheath tumors
}

\author{
Rashmi Kumari ${ }^{1, *}$, Martin R. Silic ${ }^{1, *}$, Yava L. Jones-Hall ${ }^{1,2}$, Alexandra Nin-Velez ${ }^{1}$, \\ Jer-Yen Yang ${ }^{3,4,5}$, Suresh K. Mittal ${ }^{1,2,4}$ and GuangJun Zhang ${ }^{1,2,4,5}$ \\ ${ }^{1}$ Department of Comparative Pathobiology, Purdue University, West Lafayette, Indiana 47907, USA \\ ${ }^{2}$ Purdue Institute for Inflammation, Immunology and Infectious Disease (PI4D), Purdue University, West Lafayette, Indiana \\ 47907, USA \\ ${ }^{3}$ Department of Basic Medical Sciences, Purdue University, West Lafayette, Indiana 47907, USA \\ ${ }^{4}$ Purdue University Center for Cancer Research, Purdue University, West Lafayette, Indiana 47907, USA \\ ${ }^{5}$ Purude Institute for Integrative Neuroscience, Purdue University, West Lafayette, Indiana 47907, USA \\ "These authors contributed equally to this work \\ Correspondence to: GuangJun Zhang, email: gjzhang@purdue.edu
}

Keywords: RECK; tumor suppressor gene (TSG); MPNST; PPNET; zebrafish

Abbreviations: MPNST: malignant peripheral nerve sheath tumor; PPNET: peripheral primitive neuro-ectodermal tumor; TSG: tumor suppressor gene; CNAs: copy number alterations; LOH: loss of heterozygosity

Received: May 16, $2017 \quad$ Accepted: April 08, $2018 \quad$ Published: May 04, 2018

Copyright: Kumari et al. This is an open-access article distributed under the terms of the Creative Commons Attribution License 3.0 (CC BY 3.0), which permits unrestricted use, distribution, and reproduction in any medium, provided the original author and source are credited.

\section{ABSTRACT}

Malignant peripheral nerve sheath tumors (MPNSTs) are a type of sarcoma with poor prognosis due to their complex genetic changes, invasive growth, and insensitivity to chemo- and radiotherapies. One of the most frequently lost chromosome arms in human MPNSTs is chromosome 9p. However, the cancer driver genes located on it remain largely unknown, except the tumor suppressor gene, p16 (INK4)/CDKN2A. Previously, we identified RECK as a tumor suppressor gene candidate on chromosome 9p using zebrafish-human comparative oncogenomics. In this study, we investigated the tumorigenesis of the reck gene using zebrafish genetic models in both tp53 and ribosomal protein gene mutation background. We also examined the biological effects of RECK gene restoration in human MPNST cell lines. These results provide the first genetic evidence that reck is a bona fide tumor suppressor gene for MPNSTs in zebrafish. In addition, restoration of the RECK gene in human MPNST cells leads to growth inhibition suggesting that the reactivation of RECK could serve as a potential therapeutic strategy for MPNSTs.

\section{INTRODUCTION}

Malignant peripheral nerve sheath tumors (MPNSTs) are a type of sarcoma that originate from the neural crest/Schwann cell lineage in humans, and have a poor prognosis due to their complex genetic changes, invasive growth nature, and insensitiveness to chemo- and radiotherapies [1-3]. For the etiology of MPNST, only a few cancer genes are currently known to be related to this type of malignancy: NF1, NF2, SMARCB1 and LZTR1
$[4,5]$. Surgical resection is the mainstay of treatment, but often compromised by many factors such as distant metastasis, local damage, and inoperable locations [6]. Moreover, there is currently no targeted therapy available for this type of malignancy, mainly due to the lack of knowledge of its cancer driver genes.

Cancer driver genes are a small number of genes found mutated in cancer genomes, and their mutations biologically contribute to cancer initiation and progression [7]. The remaining mutated genes in the cancer genome 
are defined as passenger genes, if their mutations have no impact on tumorigenesis. Based on gain or loss of function, cancer driver genes can be divided into either oncogenes or tumor suppressor genes (TSGs). One of the goals of current cancer research is to identify all the cancer driver genes, so that they can be pursued as new targets for the development of cancer therapy. Targeted cancer therapy has emerged as a better alternative to other treatment options because of its high specificity and fewer side effects. Effective targeted cancer therapies require accurate identification of the targetable cancer driver genes.

Identification of cancer driver genes is difficult, due to the plethora of mutations in the cancer cell genomes and the time required for functional validation. One of the extreme situations is represented with aneuploid chromosomes, which are found in more than $90 \%$ of solid tumors and $75 \%$ of hematopoietic tumors [8]. This difficulty stems from the large number of genes on the chromosome arm. Even if the driver genes have no mutations, it is not possible to distinguish driver from passenger genes despite advances in modern technologies, such as parallel sequencing and microarray. Cross-species comparative oncogenomics emerged as a solution to pinpoint cancer driver candidates, as the cancer driver genes tend to be enriched in common genetic mutations, due to the gene functional conservation. This approach has already been demonstrated to be effective in mice and dogs [9-12]. The research community has recently showed that zebrafish-human comparative oncogenomics is particularly effective with large aneuploid chromosomes because gene synteny was extensively reorganized during vertebrate evolution [13-15]. Furthermore, the zebrafish model provides a valuable system for functional studies of candidate drivers from omics studies. It has been well-established that zebrafish tumors share many similarities with humans including histology, etiology of genetic mutations (tp53, pten, etc.), the transcriptome, and copy number alterations (CNAs) [16, 17].

Chromosome $9 \mathrm{p}$ is one of the most frequently lost chromosome arms in human MPNSTs and many other types of solid tumors [18-24]. CDKN2A/p16/INK4 is one of the currently known TSGs on this chromosome arm, but this does not exclude the existence of other TSGs on 9p. Using zebrafish-human comparative oncogenomics, on human chromosome 9p, we have identified the RECK gene as a cancer driver candidate that could potentially be a tumor suppressor gene for MPNSTs [13]. RECK is located on chromosome $9 \mathrm{p}$, which is frequently lost in human MPNSTs [25]. The RECK (reversion-inducing cysteine-rich protein with Kazal motifs) gene encodes a $110 \mathrm{kDa}$ membrane-bound glycoprotein [26]. It was discovered as a gene that was able to rescue the aberrant cell morphology of RAS-transformed fibroblasts [27]. The Reck gene is expressed in many mouse tissues, and it was found to be down-regulated in many types of human cancers such as the liver, pancreatic, breast, colon, lung, melanoma, and fibrosarcoma $[26,28]$. Low expression of
RECK was reported to correspond to biological malignancy, such as invasiveness and metastasis [26, 29, 30]. Thus, the RECK gene was believed to be a tumor suppressor gene, but in vivo experimental evidence was missing to support this hypothesis. Knockout mice indicate that Reck is a developmentally essential gene and is critical for angiogenesis through regulating matrix metalloproteinases [31]. These mouse embryos die at stage E10.5 in the homozygous knockouts, due to hemorrhage and neuronal defects [31]. Moreover, there is no report of spontaneous tumors in the heterozygous RECK knockout mice. Whether the Reck gene functions as a TSG in vivo remains unknown, although it is worth noting that evidence from cell culture models uphold this hypothesis [26].

Here, we performed functional genetic studies on the zebrafish reck gene, and provided the first evidence that zebrafish reck is a tumor suppressor gene in vivo.

\section{RESULTS}

Zebrafish are becoming a popular cancer model, due to their tractable genetics and conserved vertebrate biology similar to humans [16]. In zebrafish, MPNSTs can be initiated with tp53 homozygous point mutations [32], or heterozygous ribosomal protein gene $(r p)$ mutations [33]. Previously, we have found that zebrafish and human MPSNTs share high similarities, such as histology, aneuploidy, and copy number alterations [13, 14, 32, 33]. To investigate whether $R E C K$ is a bona fide tumor suppressor gene in MPNSTs, we took advantage of the zebrafish genetic model. First, we re-analyzed our previous DNA copy number data from both zebrafish and human MPNSTs [13]. In both human and zebrafish MPNSTs, about $39 \%$ of tumors lose DNA copy numbers of the RECK gene (Figure 1A-1B). As the zebrafish genome usually contains duplicates for some of the genes, due to teleost whole genome duplication, we performed a BLAT search using the human RECK gene as a bait in both NCBI and Ensembl. Both searches suggested that a single copy of the reck gene is present in the zebrafish genome. The zebrafish Reck shares $65 \%$ protein sequence similarity with human RECK, both proteins are coded by 21 exons (Figure 1C), and both feature a KAZAL_FS (Kazal type serine protease inhibitor and follistatin-like) domain. To further demonstrate the zebrafish reck gene identity, we conducted phylogenetic analysis using protein sequences from representative species. Our maximum likelihood phylogenetic analysis revealed that $R E C K$ is a conserved gene in metazoans (Figure 1D), indicating that zebrafish reck may have similar biological functions with the human $R E C K$ gene.

The zebrafish reck gene is found to be expressed in the developing neural crest cells, and is required for appropriate sensory neuron formation in a cell-autonomous fashion [34]. Since MPNST is a type of malignancy of Schwann cells that originate from the neural crest, we first validated the expression of the zebrafish reck gene by In Situ hybridization 


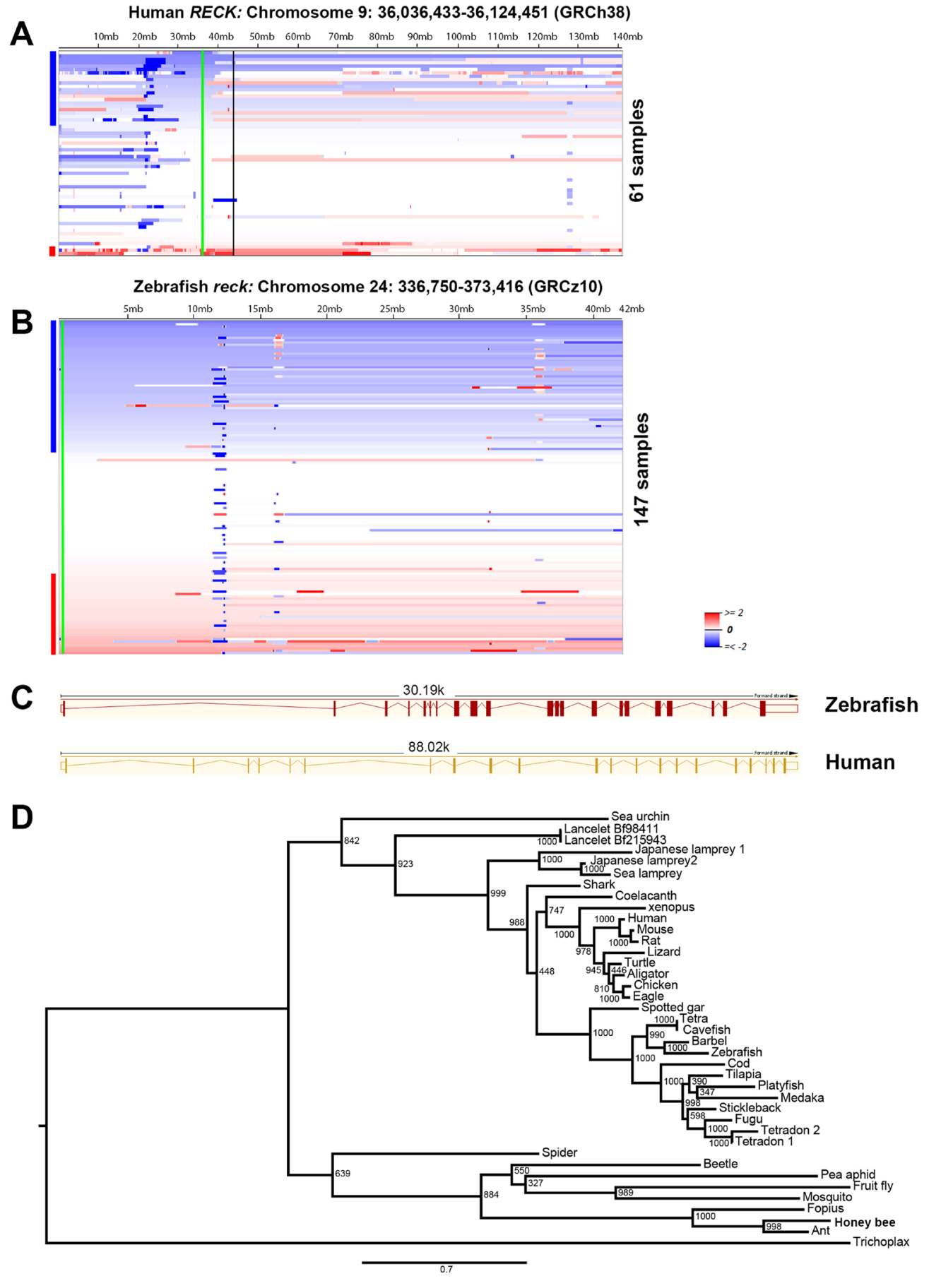

Figure 1: Frequent loss of DNA copy numbers of the $\boldsymbol{R E C K}$ gene in human and zebrafish MPNSTs. (A) Heat map of human chromosome 9 is shown. RECK is located on chromosome 9p, which is found to be under-represented in 39\% (24 out of 61) of human MPNSTs [13]. The green line represents the RECK gene locus, and the vertical black line indicates the centromere of chromosome 9. The human RECK gene location and reference genome assembly are labeled above the heat map. (B) Zebrafish reck is located on chromosome 24, which is under-represented in about 39\% (58 out of 147) of zebrafish MPNSTs. Samples are sorted top-to-bottom by decreasing deletion amplitude at their respective $R E C K /$ reck locus, indicated by a green line. Blue and red bars at the right side of each panel indicate samples with $R E C K /$ reck, losses (blue) or gains (red). Color densities are corresponding to the degree of loss and gain as previously described [13]. The zebrafish reck gene location and reference genome assembly are labeled above the heat map. (C) Human and zebrafish RECK have different intron-exon structures, but both are composed of 21 exons. The solid red/orange vertical boxes represent the gene coding regions, and the empty boxes indicate un-transcribed regions. Red/orange thin lines indicate the introns. Human RECK transcript: RECK001 ENST00000377966.3. Zebrafish reck transcript: reck-001 ENSDART00000129135.2. (D) Maximum likelihood phylogeny of RECK proteins, as obtained with JTT plus gamma distances $(\alpha=0.941)$. Numbers around each node indicate bootstrap values based on 1,000 replicates. Branch lengths are proportional to expected replacements per site. The tree was rooted with placozoa (Trichoplax adhaerens). 
during zebrafish embryogenesis. Indeed, as reported, we confirmed that reck is expressed in the developing neural crest cells (Supplementary Figure 1), suggesting it has a developmental role in the neural crest-derived cells.

A TSG is defined as a gene whose inactivation leads to tumor formation, and promotes tumor development in vivo. To functionally evaluate whether reck is a bona fide TSG in zebrafish MPNSTs, we took advantage of the currently available reck loss-of-function mutant, sensory deprived ( $s d p)$ [34]. If a TSG is strong enough, we can expect the development of spontaneous tumors in the loss-of-function mutant animals in a relatively short time. However, if a TSG is weak, we may need a longer follow-up time for tumorigenesis requiring a large number of animals. Based on the principle of genetic synthetic/ additive effect, we hypothesized that loss of reck will accelerate tumorigenesis in a tp53 or ribosomal protein mutant background, as both types of mutation were known to cause spontaneous MPNSTs in zebrafish $[32,33]$.

To test this hypothesis, we crossed the reck mutant, $s p d^{w 12 /+}$, with the tp53 or ribosomal protein L35 (rpL35) mutant, and tracked the development of tumors. In zebrafish, $17 r p$ gene mutants were known to cause MPNSTs and the impact of these genetic mutations was believed to be similar $[33,35,36]$. Here, we chose the rpL35 mutant, hi258, as a representative of the $r p$ TSGs. As expected, the reck mutation accelerated tumor formation rate in both mutant backgrounds (Figure 2A-2B), demonstrating that reck is indeed a bona fide TSG. Interestingly, we noticed that there was a tumor spectrum shift in the reck and $r p L 35$ fish cross (Figure 2C). Although most tumors were MPNSTs (Figure 2D-2E), 25\% (6 out of 24) of tumors were peripheral primitive neuroectodermal tumors (pPNETs, Figure $2 \mathrm{~F}-2 \mathrm{G}$ ) in $s d p^{w 12 /+} ; r p L 35^{h i 258 /+}$, while only $7 \%$ (2 out of 28) were pPNETs in $r p L 35^{\text {hi258/+ }}$, suggesting that the reck gene may have a cell type differential role during tumor formation. As we did not detect any visible tumors in the reck heterozygotes $\left(s d p^{w 12 /+}\right)$ in either cross, we reasoned that reck may function as a TSG in a two-hit paradigm manner, exemplified by the $R B 1$ gene [37]. To examine this possibility, we investigated the loss of heterozygosity $(\mathrm{LOH})$ in a few tumor-tail paired tissue samples. Indeed, we found 4 out of 8 cases where the wildtype allele was lost in these tumors (Figure $2 \mathrm{H}$ ). Altogether, our results supported the hypothesis that reck is a TSG that may follow the two-hit paradigm of tumor suppression.

Since the DNA copy number of $R E C K$ is frequently lost in human MPNSTs, we hypothesized that protein levels of RECK in human MPNST cell lines are generally decreased. To test this, we performed Western blots and found that RECK was not detectable or expressed at a lower level in human MPNST cells, compared to the benign neurofibroma cell line, HEI193 (Figure 3A). TSG restoration was found to inhibit tumor growth and reverse other cancer characteristics [38], therefore, we reasoned that restoration of $R E C K$ in human MPNST cells would inhibit cell growth. To demonstrate this, we first generated three tetracycline-inducible stable human MPNST cell lines using an untagged full-length RECK (pSLIK-neo$R E C K$ ) construct (Figure 3B). We chose STS26T (TP53 mutant), S462 (NF1 mutant), and 90-8TL (NF1 microdeletion) human MPNST cell lines, as TP53 and NF1 are commonly mutated in human MPNSTs $[39,40]$. Moreover, we previously found that doxycycline has no impact on the growth of these cell lines [41]. Next, we examined cell growth by MTT, colony formation assays, and soft agar anchorage-independent growth assays. Indeed, upon restoration of RECK expression the cell growth rates were decreased in all three MPNST cell lines (Figure 3C, 3F, 3I). Similarly, growth inhibition effects were also observed in the colony formation assay (Figure 3D, 3G, 3J) and the soft agar anchorage-independent growth assay (Figure $3 \mathrm{E}, 3 \mathrm{H}, 3 \mathrm{~K}$ ), suggesting that $R E C K$ functions as a TSG in human MPNST cells. Notably, this growth inhibition is not dependent on TP53 or NF1, suggesting that RECK is a mechanistically independent TSG.

\section{DISCUSSION}

The RECK gene is one of the TSG candidates on human chromosome $9 \mathrm{p}$ in MPNSTs and other types of tumors [18-24]. Although, accumulating evidence from cellular experiments indicates that the RECK gene is a TSG in vivo, there is no clear genetic evidence to prove this notion, since this gene is a developmentally essential gene in mouse $[26,31]$. Here, our results provide the first in vivo evidence that reck is a bona fide tumor suppressor gene in zebrafish MPNSTs. Moreover, we validate that the restoration of RECK leads to inhibition of human MPNST cell growth.

In our genetic experiments, the loss of one reck gene allele indeed accelerated tumorigenesis initiated with tp53-/-, or $r p L 35^{\text {hi258/+ }}$, although we did not find spontaneous tumors in zebrafish reck mutant, $s d p^{w 12 /+}$, within 2 years. These genetic synthetic/additive effects between reck and tp53 or rpL35 suggested that reck is a TSG and it is not in the same genetic pathway of tp53 or $r p L 35$, i.e. there is no direct genetic interactions. It is worth to note that the heterozygous recksdpw12/+ alone did not lead to any visible tumors in our studies, indicating that $\mathrm{LOH}$ is required for reck tumorigenicity, or that reck is a weaker haploinsufficient TSG, compared to tp53 or rpL35 in zebrafish. TSGs usually function through LOH (two wildtype alleles are inactivated) or haploinsufficiency (one wildtype allele is inactivated) [37]. Half of our examined tumor samples (4 out 8) showed loss of the wildtype allele, suggesting that the reck gene may fit $\mathrm{LOH}$, and it likely follows the two-hit paradigm of tumor suppression in zebrafish. However, we cannot exclude the possibility of the haploinsufficiency mechanism, since we were only able to examine 8 samples. Further studies with more 
tumors samples will be needed to address this question, and whether human RECK follows the same rule.

One of the interesting observations is that the tumor spectrum shifts in the reck mutant zebrafish. Generally, tp53 or rpL35 mutations lead to MPNSTs in zebrafish $[32,33]$. In this study, $25 \%$ (6 out of 24 ) of tumors were
pPNETs in $s d p^{w 12 /+} ; r p L 35^{h i 258 /+}$, while only $7 \%$ (2 out of 28 ) were pPNETs in $r p L 35^{\text {hi258/+ }}$. A pPNET is a type of round cell sarcoma belonging to the Ewing family of tumors, which also originate from the neural ectoderm where neural crest cells develop [42]. Considering a potential developmental role of reck in the zebrafish neural crest
A

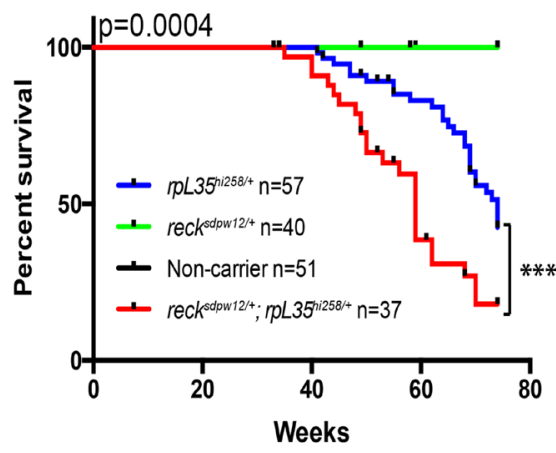

B

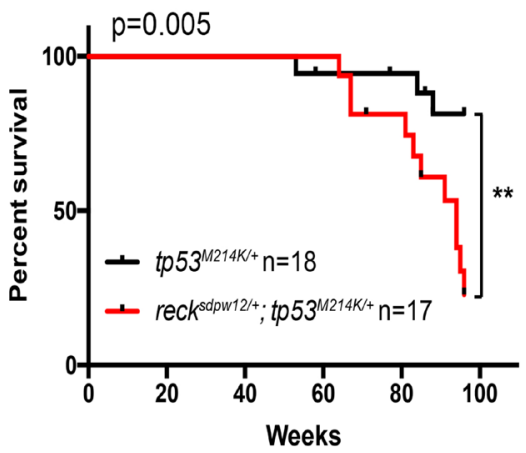

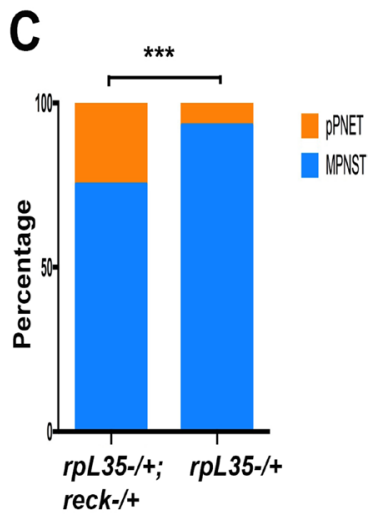
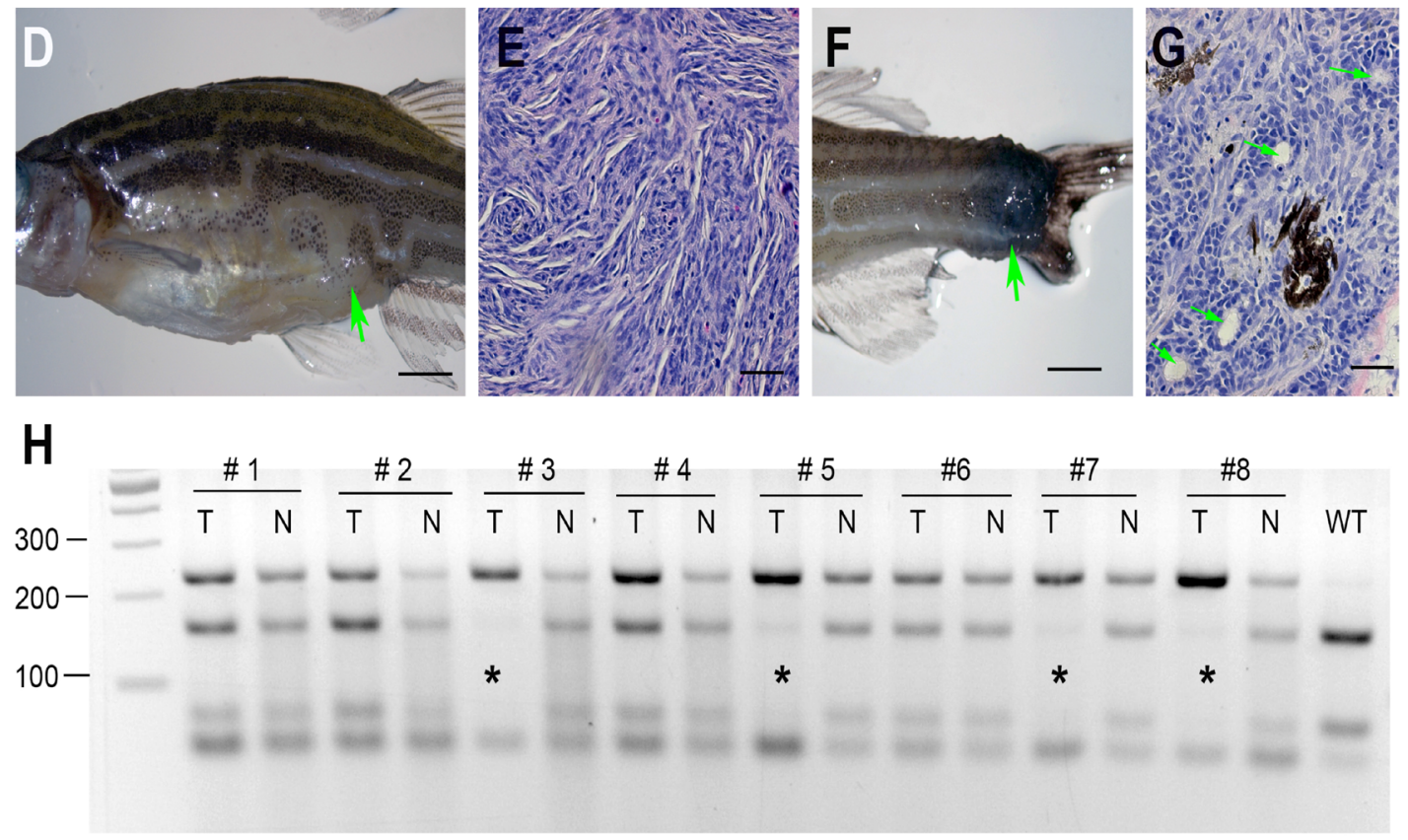

Figure 2: Reck is a tumor suppressor gene in zebrafish MPNSTs. (A-B) Loss of the reck gene function facilitates tumorigenesis in either $r p$ or tp53 mutant background. Kaplan-Meier curves showing tumor-free survival of cohorts of single and double heterozygotes derived from $r p L 35^{h i 258 /+} \times s d p^{w 12 /+}$ crosses (A) and $t p 53^{M 214 K /+} \times s d p^{w 12 /+}$ crosses (B). Fish from all crosses were genotyped by PCR for each relevant mutation at 6-8 weeks of age, and housed segregated by genotype. In all panels, the numbers of fish (n) of each genotype are shown next to the genotype. The $\mathrm{p}$ values between the $r p$ or tp53 single heterozygote and the compound mutants are shown in the figures. The green line $\left(s d p^{w 12 /+}\right)$ and black line (non-carrier) are overlapped in panel (A). (C) More zebrafish developed pPNETs in the $r p L 35^{h i 258 /+} ; s d p^{w 12 /+}$ compared to $r p L 35^{\text {hi258/+}}$. Two-tailed fisher's exact test was used for statistical analysis. $p=0.008$. Asterisks indicate statistical significance (A-C). (D) A typical zebrafish with abdominal MNPST. The green arrow indicates the position of the tumor. (E) Hematoxylin and Eosin (H\&E) staining showed typical histopathology of zebrafish MPNST, which is characterized by interlacing bundles and swirls of spindle shaped tumor cells. (F) A typical pPNET that was found in the tail region. The green arrow indicates the position of the tumor. (G) Representative examples of pPNET histology by H\&E staining. The pseudorosettes (Flexner-Wintersteiner rosettes) are indicated with green arrows. (H) The wildtype allele is lost in some of the $t p 53^{M 214 K /+} ; s d p^{w 12 /+}$ tumors (tumor samples are labeled with asterisks). The reck gene was amplified by PCR using primers flanking the $s d p^{w 12}$ mutation site. The PCR product was then digested with Ava II, which only cuts the $233 \mathrm{bps}$ wildtype PCR product into $158 \mathrm{bps}$ and $75 \mathrm{bps}$ fragments [34]. T, tumor; N, normal tissue from caudal fin. Note, the lowest band is from the PCR primer polymerization. Scaler bar $=250 \mathrm{~mm}(\mathrm{D}, \mathrm{F}) \mathrm{Scale}$ bar $=50 \mathrm{~mm}(\mathrm{E}, \mathrm{G})$. 
(reported previously [34] and Supplementary Figure 1), the loss of reck function is likely responsible for the tumor cell differentiation to pPNETs instead of MPNSTs. RECK was recently found to regulate WNT $[43,44]$ and NOTCH [45] signaling pathways, which are important for neural crest differentiation $[46,47]$ and MPNST tumorigenesis [48-50]. Future studies are required to reveal the detailed mechanism behind this phenomenon.
Identification of new TSGs is not only important for the understanding of cancer biology, but also the key for cancer targeted therapy. Cancer targeted therapy is generally believed to be aimed at an oncogene. However, TSGs are also important for cancer targeted therapy, since the discovery of synthetic lethality of the PARPI and $B R C A 1 / 2$ genes and their clinical applications using PARP inhibitors [51, 52]. Along the same direction,
A

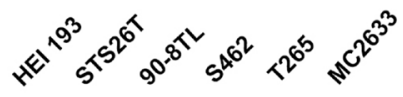

RECK

ACTB

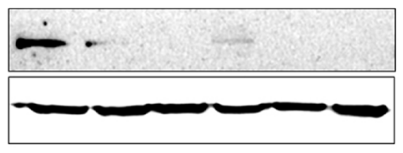

C

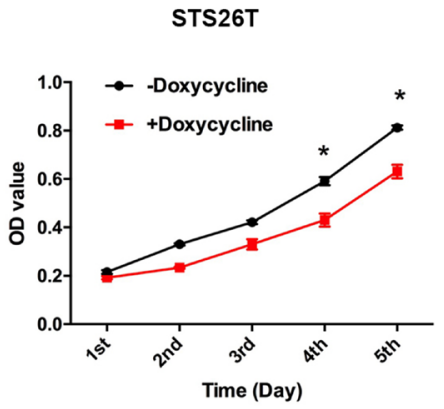

$\mathbf{F}$

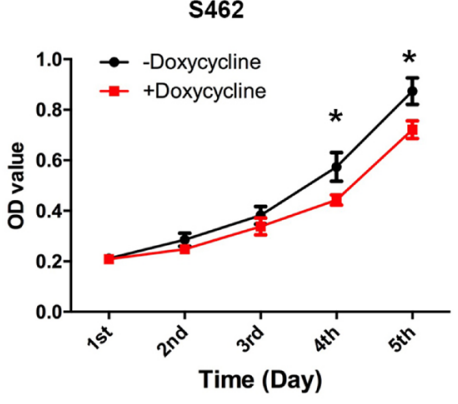

I

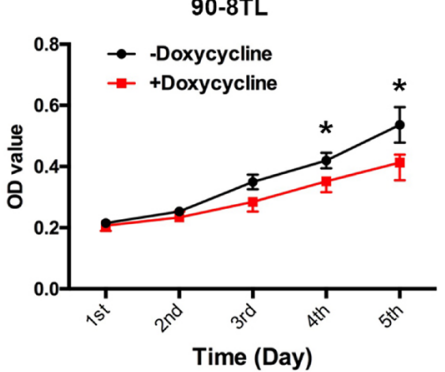

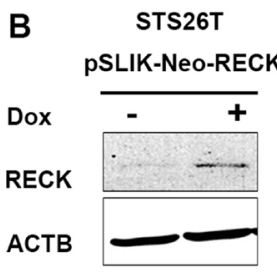

STS26T

D
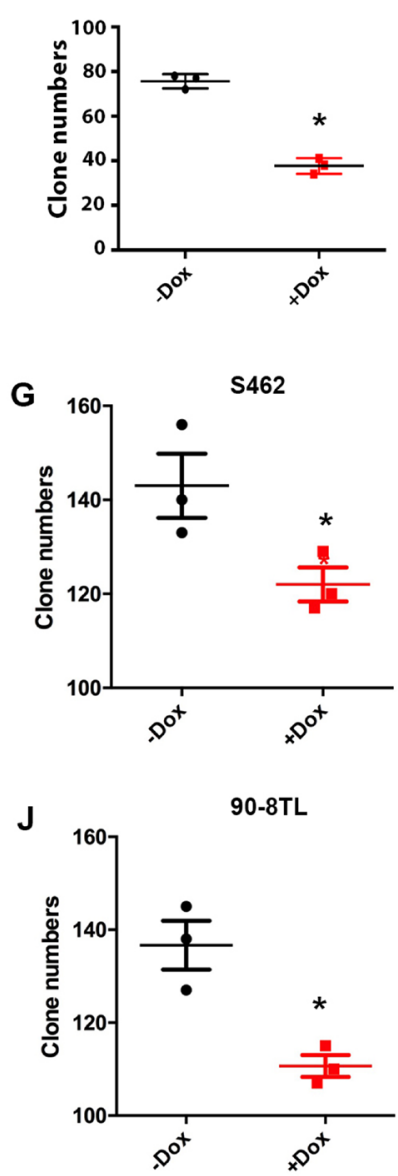

S462

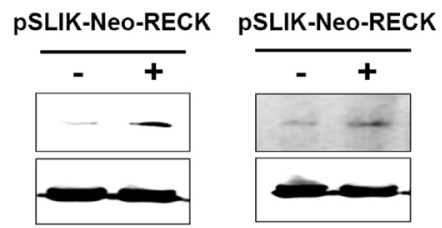

E

STS26T
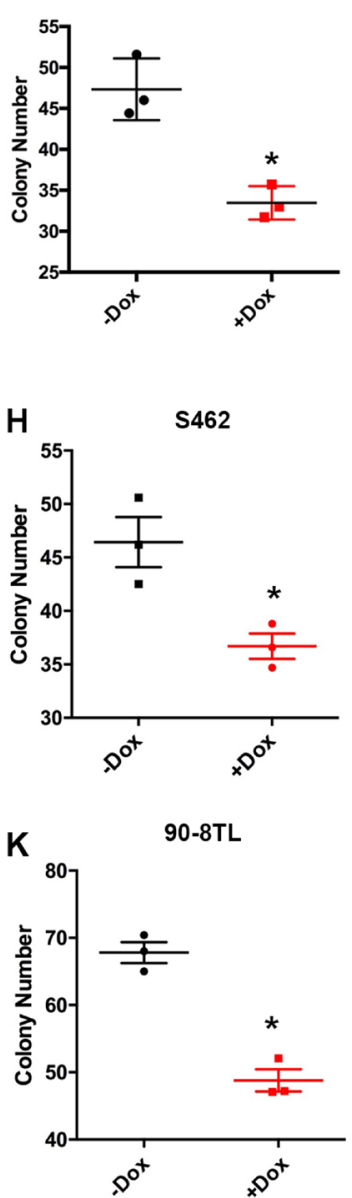

Figure 3: Restoration of $\boldsymbol{R E C K}$ in human MPNST cells suppresses cell growth. (A) RECK expression is lost or dramatically decreased in human MPNST cells (STS26T, 90-8TL, S462, T265T and MC2633) compared to a benign neurofibroma cell line, HEI193. (B) RECK expression was restored in lentiviral RECK-stable cells (STS26T, S462 and 90-8TL) induced by doxycycline (1 $\mu \mathrm{g} / \mathrm{ml}) \mathrm{shown}$ by Western blots. (C, F, I) MTT assays revealed that cell growth was inhibited by RECK restoration in STS26T, S462 and 90-8TL cell lines, respectively. Growth curves were created using the optical density values collected over five days. (D, G, J) Independent cell growth by plate colony formation assays on STS26T, S462 and 90-8TL, respectively. Crystal violet stained cell colonies (diameter $\geq 0.2 \mathrm{~mm}$ ) were counted. Asterisk indicated the statistical significance by student $t$-test, $p<0.05$. (E, H, K) Anchorage- independent growth by soft agar assay. The cells were seeded on agarose plates for colony formation assays, and colonies whose diameters are larger than $20 \mathrm{um}$ were counted two weeks later. Asterisks indicated the statistical significance by student $t$-test, $p<0.05$. 
TSG restoration was also demonstrated as another way of targeted therapy based on the biology of TSGs [53]. In the case of the $R E C K$ gene, it is frequently reported to be silenced through promoter methylation in a variety of human cancers [54-59]. Thus, attempts were made to restore RECK expression using DNA methyltransferase (DNMT) inhibitor, 5-aza-2'deoxycytidine (5-aza-dC), in colon cancer and salivary adenoid cystic carcinoma $[60,61]$. Our cellular experiments on RECK restoration also support the possibility of RECK as a target for MPNST therapy. Furthermore, cancer driver genes that are located on the same chromosome region were found to cooperate and promote tumorigenesis [62]. The discovery of such combinations is very important in developing new cancer therapies. For example, this phenomenon has recently been reported in human colon cancers, where the TP53 copy number loss was coupled with the loss of a neighboring gene, POLR2A [63]. Identification of other TSGs on the chromosome $9 \mathrm{p}$ might further help to understand the tumorigenic mechanism of RECK and to develop new cancer treatment strategies based on the concept of gene cooperation.

\section{MATERIALS AND METHODS}

\section{Zebrafish lines, husbandry, and tumor onset analysis}

Zebrafish were raised and maintained at the Purdue animal housing facility, which is approved by AAALAC. All experiments were carried out according to the protocols approved by the Purdue Animal Care and Use Committee (PACUC). The tumor-prone zebrafish lines carrying either the $t p 53^{M 214 K / M 214 K}$ point mutation, or insertional mutations in ribosomal protein gene, $r p L 35^{\text {hi258/+ }}$, have been described previously [32, 33]. The zebrafish reck point mutation carrier, $s d p^{w 12 /+}$, was acquired from the laboratory of Dr. David W. Raible at the University of Washington. After crossing, genotyping was carried out at 6-8 weeks of age using previously published genotyping methods $[13,34]$.

After genotyping, siblings of different genotypes were housed in adjacent tanks at similar densities to minimize environmental differences. From the sixth month, we started to check for fish with visible tumors. During tumorigenesis, fish were euthanized at first observation of tumors or other signs of illness. Any accidental fish loss was censored. The tumor tissue samples from the euthanized fish were processed for hematoxylin and eosin (H\&E) staining. The tumor types were confirmed by a board certified veterinary pathologist, blinded to the experimental groups. The final KaplanMeier tumor free survival curves were generated, and log-rank tests were performed to determine statistical significance using GraphPad Prism 6.0 h [13].

\section{RECK protein sequence and phylogeny analyses}

RECK protein sequences were identified by a BLASTp search using the human RECK sequence as a query in Ensembl, NCBI, or JGI website. The longest sequence was preferentially chosen when there were multiple sequences. Multiple protein sequences (Supplementary Table 1) were aligned using MUSLE program [64], and the FASTA format alignment can be found in the Supplementary File 1. The evolutionary model for phylogenetic analysis was identified using a best model test using maximum likelihood, and default parameters in MEGA6 [65]. A maximum likelihood phylogenetic tree was constructed using JTT $+\mathrm{G}$ with 1000 bootstrap replicates with PhyML 3.1 [66]. The final phylogenetic trees were viewed and generated with FigTree V1.4.2 (http://tree.bio.ed.ac.uk/software/figtree). Gene intron-exon structures were analyzed using the longest transcripts in Ensembl.

\section{Cell culture, stable cell lines, and cell growth assays}

All experimental protocols using cell lines and plasmid constructs were approved by Purdue University institutional review board. The human MPNST cell lines were authenticated by ATCC using short tandem repeat profiling (April 25, 2016). Cells were cultured in DMEM with $10 \%$ heat inactivated fetal bovine serum, penicillin (100 IU/ml), and streptomycin $(100 \mu \mathrm{g} / \mathrm{ml})$. All cell cultures were carried out at $37^{\circ} \mathrm{C}$ in a humidified $5 \%$ $\mathrm{CO}_{2}$ atmosphere. A full-length sequence of the $R E C K$ gene was amplified from HEK293T cells, and sequences were confirmed by Sanger sequencing. The RECK gene was subsequently cloned into pSlik-Neo vector (Addgene \#25735). Stable cell establishment, MTT and plate colony assays were conducted following previously published methods [41]. For Western blot, commercial anti-RECK antibody (BD, \#611512 or Cell Signaling \#3433, 1:500 dilution) was used following an established method [41]. Statistical analyses were performed using GraphPad Prism $6.0 \mathrm{~h}$. Data were analyzed using the un-paired student $t$-test. $p<0.05$ was considered as statistically significant.

\section{Soft agar assay}

For each cell line, 1000 cells per well were seeded in six-well plates. $1.5 \mathrm{ml} \mathrm{1 \%}$ agarose was plated at the bottom of plate. After solidification, $1 \mathrm{ml}$ of single-cell suspension in $0.7 \%$ agarose in DMEM supplemented with $10 \%$ FBS were added in each well. Then, the plates were incubated at $37^{\circ} \mathrm{C}$ in a humidified incubator with $5 \% \mathrm{CO}_{2}$. On the second day, doxycycline $(1 \mu \mathrm{g} / \mathrm{ml})$ or PBS (phosphatebuffered saline) were added to induce RECK expression. The medium was refreshed every 2 days to maintain 
constant expression of RECK. Two weeks later, cells were stained with $0.01 \%$ crystal violet solution. After multiple washes with PBS, the cell colonies were visualized and imaged under a bright field microscope. Six sub-images at different focus in the same field were captured at $40 \times$ magnification, and all the images of the same field were $\mathrm{Z}$ stacked using Image $\mathrm{J}$ software. Ten random bright field images of each sample were captured. Total number of formed foci (diameter $>20 \mu \mathrm{m}$ ) in each field were counted manually in Image $\mathrm{J}$ software. Three technical replicates were included, and each experiment was repeated at least three times for each experimental condition.

\section{In situ hybridization}

The primers Dr.reck-97F (5' CACCATGAGCGG GTGTCTCCAGATCCTCA3') and Dr.reck-2961R (5' GAGGTCAGAGGTCAGGGTCAGGAT3') were used for the zebrafish reck gene cloning PCR. The zebrafish reck gene cloning and In Situ hybridization were performed using the standard methods as previously described [67].

\section{Author contributions}

GZ designed the project and coordinated research; RK, MRS, ANV, and GZ performed experiments; RK, MRS, YLJ, JYY, SKM, and GZ analyzed the results; GZ wrote the original draft. MRS, RK, YLJ, JYY, SKM, and GZ reviewed and edited the manuscript. All authors read and approved the final manuscript.

\section{ACKNOWLEDGMENTS AND FUNDING}

We gratefully acknowledge the Heyward Foundation for supporting our research. We thank Dr. David W. Raible for sharing the $s d p^{w 12}$ fish. We also thank Dr. George De Vries, and Dr. Karen Cichowski for kindly providing us the MPNST cell lines. We thank Guolin Chai and Xinmin Yan for technical help generating the pSlik-RECK construct, and Zhibin Cui and Pei-Chieh Tien for cell line and antibody validations. The authors also thank the support from the Purdue University Center for Cancer Research, NIH grant P30 CA023168, and Jim and Diann Robbers Cancer Research Grant for New Investigators. We thank the Purdue Histology Research Laboratory for preparation of tissue sections.

\section{CONFLICTS OF INTEREST}

None.

\section{REFERENCES}

1. Ducatman BS, Scheithauer BW, Piepgras DG, Reiman HM, Ilstrup DM. Malignant peripheral nerve sheath tumors.
A clinicopathologic study of 120 cases. Cancer. 1986; 57:2006-21.

2. Kolberg M, Holand M, Agesen TH, Brekke HR, Liestol K, Hall KS, Mertens F, Picci P, Smeland S, Lothe RA. Survival meta-analyses for $>1800$ malignant peripheral nerve sheath tumor patients with and without neurofibromatosis type 1 . Neuro Oncol. 2013; 15:135-47. https://doi.org/10.1093/ neuonc/nos 287 .

3. Durbin AD, Ki DH, He S, Look AT. Malignant Peripheral Nerve Sheath Tumors. Adv Exp Med Biol. 2016; 916:495530. https://doi.org/10.1007/978-3-319-30654-4_22.

4. Piotrowski A, Xie J, Liu YF, Poplawski AB, Gomes AR, Madanecki P, Fu C, Crowley MR, Crossman DK, Armstrong L, Babovic-Vuksanovic D, Bergner A, Blakeley JO, et al. Germline loss-of-function mutations in LZTR1 predispose to an inherited disorder of multiple schwannomas. Nat Genet. 2014; 46:182-87. https://doi.org/10.1038/ng.2855.

5. McClatchey AI. Neurofibromatosis. Annu Rev Pathol. 2007; 2:191-216. https://doi.org/10.1146/annurev. pathol.2.010506.091940.

6. Farid M, Demicco EG, Garcia R, Ahn L, Merola PR, Cioffi A, Maki RG. Malignant peripheral nerve sheath tumors. Oncologist. 2014; 19:193-201. https://doi.org/10.1634/ theoncologist.2013-0328.

7. Stratton MR, Campbell PJ, Futreal PA. The cancer genome. Nature. 2009; 458:719-24. https://doi.org/10.1038/nature07943.

8. Weaver BA, Cleveland DW. Does aneuploidy cause cancer? Curr Opin Cell Biol. 2006; 18:658-67.

9. Kim M, Gans JD, Nogueira C, Wang A, Paik JH, Feng B, Brennan C, Hahn WC, Cordon-Cardo C, Wagner SN, Flotte TJ, Duncan LM, Granter SR, et al. Comparative oncogenomics identifies NEDD9 as a melanoma metastasis gene. Cell. 2006; 125:1269-81. https://doi.org/10.1016/j.cell.2006.06.008.

10. Maser RS, Choudhury B, Campbell PJ, Feng B, Wong KK, Protopopov A, O’Neil J, Gutierrez A, Ivanova E, Perna I, Lin E, Mani V, Jiang S, et al. Chromosomally unstable mouse tumours have genomic alterations similar to diverse human cancers. Nature. 2007; 447:966-71.

11. Zender L, Spector MS, Xue W, Flemming P, CordonCardo C, Silke J, Fan ST, Luk JM, Wigler M, Hannon GJ, $\mathrm{Mu} \mathrm{D}$, Lucito $\mathrm{R}$, Powers $\mathrm{S}$, et al. Identification and validation of oncogenes in liver cancer using an integrative oncogenomic approach. Cell. 2006; 125:1253-67. https:// doi.org/10.1016/j.cell.2006.05.030.

12. Tang J, Le S, Sun L, Yan X, Zhang M, Macleod J, Leroy B, Northrup N, Ellis A, Yeatman TJ, Liang Y, Zwick ME, Zhao S. Copy number abnormalities in sporadic canine colorectal cancers. Genome Res. 2010; 20:341-50. https:// doi.org/10.1101/gr.092726.109.

13. Zhang G, Hoersch S, Amsterdam A, Whittaker CA, Beert E, Catchen JM, Farrington S, Postlethwait JH, Legius E, Hopkins N, Lees JA. Comparative oncogenomic analysis of copy number alterations in human and zebrafish tumors 
enables cancer driver discovery. PLoS Genet. 2013; 9:e1003734. https://doi.org/10.1371/journal.pgen.1003734.

14. Zhang G, Hoersch S, Amsterdam A, Whittaker CA, Lees JA, Hopkins N. Highly aneuploid zebrafish malignant peripheral nerve sheath tumors have genetic alterations similar to human cancers. Proc Natl Acad Sci U S A. 2010; 107:16940-5. https://doi.org/10.1073/pnas.1011548107.

15. Chen EY, Dobrinski KP, Brown KH, Clagg R, Edelman E, Ignatius MS, Chen JY, Brockmann J, Nielsen GP, Ramaswamy S, Keller C, Lee C, Langenau DM. Crossspecies array comparative genomic hybridization identifies novel oncogenic events in zebrafish and human embryonal rhabdomyosarcoma. PLoS Genet. 2013; 9:e1003727. https://doi.org/10.1371/journal.pgen.1003727.

16. White R, Rose K, Zon L. Zebrafish cancer: the state of the art and the path forward. Nat Rev Cancer. 2013; 13:624-36. https://doi.org/10.1038/nrc3589.

17. Liu S, Leach SD. Zebrafish models for cancer. Annu Rev Pathol. 2011; 6:71-93. https://doi.org/10.1146/ annurev-pathol-011110-130330.

18. Gunawan B, Schulten HJ, von Heydebreck A, Schmidt B, Enders C, Hoer J, Langer C, Schuler P, Schindler CG, Kuhlgatz J, Fuzesi L. Site-independent prognostic value of chromosome $9 \mathrm{q}$ loss in primary gastrointestinal stromal tumours. J Pathol. 2004; 202:421-9. https://doi. org/10.1002/path.1537.

19. Lichun Y, Ching Tang CM, Wai Lau K, Lung ML. Frequent loss of heterozygosity on chromosome 9 in Chinese esophageal squamous cell carcinomas. Cancer Lett. 2004; 203:71-7.

20. Van Tilborg AA, Hekman AC, Vissers KJ, van der Kwast TH, Zwarthoff EC. Loss of heterozygosity on chromosome 9 and loss of chromosome 9 copy number are separate events in the pathogenesis of transitional cell carcinoma of the bladder. Int J Cancer. 1998; 75:9-14.

21. Zhu Y, Spitz MR, Strom S, Tomlinson GE, Amos CI, Minna JD, Wu X. A case-control analysis of lymphocytic chromosome 9 aberrations in lung cancer. Int $\mathrm{J}$ Cancer. 2002; 102:536-40. https://doi.org/10.1002/ijc.10762.

22. Huntly BJ, Bench AJ, Delabesse E, Reid AG, Li J, Scott MA, Campbell L, Byrne J, Pinto E, Brizard A, Niedermeiser D, Nacheva EP, Guilhot F, et al. Derivative chromosome 9 deletions in chronic myeloid leukemia: poor prognosis is not associated with loss of ABL-BCR expression, elevated BCR-ABL levels, or karyotypic instability. Blood. 2002; 99:4547-53.

23. Eiriksdottir G, Sigurdsson A, Jonasson JG, Agnarsson BA, Sigurdsson H, Gudmundsson J, Bergthorsson JT, Barkardottir RB, Egilsson V, Ingvarsson S. Loss of heterozygosity on chromosome 9 in human breast cancer: association with clinical variables and genetic changes at other chromosome regions. Int J Cancer. 1995; 64:378-82.

24. Beroukhim R, Mermel CH, Porter D, Wei G, Raychaudhuri S, Donovan J, Barretina J, Boehm JS, Dobson J, Urashima
M, Mc Henry KT, Pinchback RM, Ligon AH, et al. The landscape of somatic copy-number alteration across human cancers. Nature. 2010; 463:899-905. https://doi. org/10.1038/nature08822.

25. Beert E, Brems H, Daniels B, De Wever I, Van Calenbergh F, Schoenaers J, Debiec-Rychter M, Gevaert O, De Raedt T, Van Den Bruel A, de Ravel T, Cichowski K, Kluwe L, et al. Atypical neurofibromas in neurofibromatosis type 1 are premalignant tumors. Genes Chromosomes Cancer. 2011; 50:1021-32. https://doi.org/10.1002/gcc.20921.

26. Noda M, Takahashi C. Recklessness as a hallmark of aggressive cancer. Cancer Sci. 2007; 98:1659-65. https:// doi.org/10.1111/j.1349-7006.2007.00588.x.

27. Takahashi C, Sheng Z, Horan TP, Kitayama H, Maki M, Hitomi K, Kitaura Y, Takai S, Sasahara RM, Horimoto A, Ikawa Y, Ratzkin BJ, Arakawa T, et al. Regulation of matrix metalloproteinase- 9 and inhibition of tumor invasion by the membrane-anchored glycoprotein RECK. Proc Natl Acad Sci U S A. 1998; 95:13221-6.

28. Alexius-Lindgren M, Andersson E, Lindstedt I, Engstrom W. The RECK gene and biological malignancy-its significance in angiogenesis and inhibition of matrix metalloproteinases. Anticancer Res. 2014; 34:3867-73.

29. Jacomasso $\mathrm{T}$, Trombetta-Lima M, Sogayar MC, Winnischofer SM. Downregulation of reversion-inducing cysteine-rich protein with Kazal motifs in malignant melanoma: inverse correlation with membrane-type 1-matrix metalloproteinase and tissue inhibitor of metalloproteinase 2. Melanoma Res. 2014; 24:32-9. https:// doi.org/10.1097/CMR.0000000000000039.

30. Span PN, Sweep CG, Manders P, Beex LV, Leppert D, Lindberg RL. Matrix metalloproteinase inhibitor reversioninducing cysteine-rich protein with Kazal motifs: a prognostic marker for good clinical outcome in human breast carcinoma. Cancer. 2003; 97:2710-5. https://doi. org/10.1002/cncr.11395.

31. Oh J, Takahashi R, Kondo S, Mizoguchi A, Adachi E, Sasahara RM, Nishimura S, Imamura Y, Kitayama H, Alexander DB, Ide C, Horan TP, Arakawa T, et al. The membrane-anchored MMP inhibitor RECK is a key regulator of extracellular matrix integrity and angiogenesis. Cell. 2001; 107:789-800.

32. Berghmans S, Murphey RD, Wienholds E, Neuberg D, Kutok JL, Fletcher CD, Morris JP, Liu TX, Schulte-Merker S, Kanki JP, Plasterk R, Zon LI, Look AT. tp53 mutant zebrafish develop malignant peripheral nerve sheath tumors. Proc Natl Acad Sci U S A. 2005; 102:407-12. https://doi. org/10.1073/pnas.0406252102.

33. Amsterdam A, Sadler KC, Lai K, Farrington S, Bronson RT, Lees JA, Hopkins N. Many ribosomal protein genes are cancer genes in zebrafish. PLoS Biol. 2004; 2:E139. https:// doi.org/10.1371/journal.pbio.0020139.

34. Prendergast A, Linbo TH, Swarts T, Ungos JM, McGraw HF, Krispin S, Weinstein BM, Raible DW. The 
metalloproteinase inhibitor Reck is essential for zebrafish DRG development. Development. 2012; 139:1141-52. https://doi.org/10.1242/dev.072439.

35. Lai K, Amsterdam A, Farrington S, Bronson RT, Hopkins N, Lees JA. Many ribosomal protein mutations are associated with growth impairment and tumor predisposition in zebrafish. Dev Dyn. 2009; 238:76-85. https://doi. org/10.1002/dvdy.21815.

36. MacInnes AW, Amsterdam A, Whittaker CA, Hopkins $\mathrm{N}$, Lees JA. Loss of p53 synthesis in zebrafish tumors with ribosomal protein gene mutations. Proc Natl Acad Sci USA. 2008; 105:10408-13. https://doi.org/10.1073/ pnas.0805036105.

37. Berger AH, Knudson AG, Pandolfi PP. A continuum model for tumour suppression. Nature. 2011; 476:163-9. https:// doi.org/10.1038/nature10275.

38. Ventura A, Kirsch DG, McLaughlin ME, Tuveson DA, Grimm J, Lintault L, Newman J, Reczek EE, Weissleder $\mathrm{R}$, Jacks T. Restoration of p53 function leads to tumour regression in vivo. Nature. 2007; 445:661-5. https://doi. org/10.1038/nature05541.

39. Miller SJ, Rangwala F, Williams J, Ackerman P, Kong S, Jegga AG, Kaiser S, Aronow BJ, Frahm S, Kluwe L, Mautner V, Upadhyaya M, Muir D, et al. Large-scale molecular comparison of human schwann cells to malignant peripheral nerve sheath tumor cell lines and tissues. Cancer Res. 2006; 66:2584-91. https://doi.org/10.1158/0008-5472.can-05-3330.

40. De Raedt T, Beert E, Pasmant E, Luscan A, Brems H, Ortonne N, Helin K, Hornick JL, Mautner V, KehrerSawatzki H, Clapp W, Bradner J, Vidaud M, et al. PRC2 loss amplifies Ras-driven transcription and confers sensitivity to BRD4-based therapies. Nature. 2014; 514:247-51. https:// doi.org/10.1038/nature13561.

41. Cui Z, Shen Y, Chen KH, Mittal SK, Yang JY, Zhang G. KANK1 inhibits cell growth by inducing apoptosis through regulating $\mathrm{CXXC5}$ in human malignant peripheral nerve sheath tumors. Sci Rep. 2017; 7:40325. https://doi. org/10.1038/srep40325.

42. Tsokos M, Alaggio RD, Dehner LP, Dickman PS. Ewing sarcoma/peripheral primitive neuroectodermal tumor and related tumors. Pediatr Dev Pathol. 2012; 15:108-26. https://doi.org/10.2350/11-08-1078-PB.1.

43. Ulrich F, Carretero-Ortega J, Menendez J, Narvaez C, Sun B, Lancaster E, Pershad V, Trzaska S, Veliz E, Kamei M, Prendergast A, Kidd KR, Shaw KM, et al. Reck enables cerebrovascular development by promoting canonical Wnt signaling. Development. 2016; 143:1055. https://doi. org/10.1242/dev.136507.

44. Cho C, Smallwood PM, Nathans J. Reck and Gpr124 Are Essential Receptor Cofactors for Wnt7a/Wnt7b-Specific Signaling in Mammalian CNS Angiogenesis and BloodBrain Barrier Regulation. Neuron. 2017; 95:1221-5. https:// doi.org/10.1016/j.neuron.2017.08.032.

45. Park S, Lee C, Sabharwal P, Zhang M, Meyers CL, Sockanathan S. GDE2 promotes neurogenesis by glycosylphosphatidylinositol-anchor cleavage of RECK. Science. 2013; 339:324-8. https://doi.org/10.1126/ science.1231921.

46. Dorsky RI, Moon RT, Raible DW. Control of neural crest cell fate by the Wnt signalling pathway. Nature. 1998; 396:370-3. https://doi.org/10.1038/24620.

47. Cornell R, Eisen J. Notch in the pathway: The roles of Notch signaling in neural crest development. Semin Cell Dev Biol. 2005; 16:663-72. https://doi.org/10.1016/j. semcdb.2005.06.009.

48. Muraguchi T, Takegami Y, Ohtsuka T, Kitajima S, Chandana EP, Omura A, Miki T, Takahashi R, Matsumoto N, Ludwig A, Noda M, Takahashi C. RECK modulates Notch signaling during cortical neurogenesis by regulating ADAM10 activity. Nat Neurosci. 2007; 10:838-45. https:// doi.org/10.1038/nn1922.

49. Noda M, Vallon M, Kuo CJ. The Wnt7's Tale: A story of an orphan who finds her tie to a famous family. Cancer Sci. 2016; 107:576-82. https://doi.org/10.1111/cas.12924.

50. Watson AL, Rahrmann EP, Moriarity BS, Choi K, Conboy CB, Greeley AD, Halfond AL, Anderson LK, Wahl BR, Keng VW, Rizzardi AE, Forster CL, Collins MH, et al. Canonical Wnt/beta-catenin Signaling Drives Human Schwann Cell Transformation, Progression, and Tumor Maintenance. Cancer Discov. 2013; 3:674-89. https://doi. org/10.1158/2159-8290.CD-13-0081.

51. Morris LG, Chan TA. Therapeutic targeting of tumor suppressor genes. Cancer. 2015; 121:1357-68. https://doi. org/10.1002/cncr.29140.

52. Wang H, Han H, Mousses S, Von Hoff DD. Targeting loss-of-function mutations in tumor-suppressor genes as a strategy for development of cancer therapeutic agents. Semin Oncol. 2006; 33:513-20. https://doi.org/10.1053/j. seminoncol.2006.04.013.

53. Xue W, Zender L, Miething C, Dickins RA, Hernando E, Krizhanovsky V, Cordon-Cardo C, Lowe SW. Senescence and tumour clearance is triggered by p53 restoration in murine liver carcinomas. Nature. 2007; 445:656-60. https:// doi.org/10.1038/nature05529.

54. Du YY, Dai DQ, Yang Z. Role of RECK methylation in gastric cancer and its clinical significance. World J Gastroenterol. 2010; 16:904-8.

55. Pesta M, Kulda V, Topolcan O, Safranek J, Vrzalova J, Cerny R, Holubec L. Significance of methylation status and the expression of RECK mRNA in lung tissue of patients with NSCLC. Anticancer Res. 2009; 29:4535-9.

56. Lu XX, Zhang SM, Fang Y, Wang ZT, Xie JJ, Zhan Q, Deng XX, Chen H, Jin JB, Peng CH, Li HW, Shen BY. Clinical significance of RECK promoter methylation in pancreatic ductal adenocarcinoma. Tumour Biol. 2013; 34:3339-43. https://doi.org/10.1007/s13277-013-0903-Z.

57. Zhang CS, Ling Y, Zhang CH, Xu Y, Gao L, Li R, Zhu J, Fan LY, Wei LX. The Silencing of RECK Gene is Associated with Promoter Hypermethylation and Poor 
Survival in Hepatocellular Carcinoma. Int J Biol Sci. 2012; 8:451-8. https://doi.org/10.7150/ijbs.4038.

58. Chang HC, Cho CY, Hung WC. Downregulation of RECK by promoter methylation correlates with lymph node metastasis in non-small cell lung cancer. Cancer Sci. 2007; 98:169-73. https://doi.org/10.1111/j.1349-7006.2006.00367.x.

59. Shi G, Yoshida Y, Yuki K, Nishimura T, Kawata Y, Kawashima M, Iwaisako K, Yoshikawa K, Kurebayashi J, Toi M, Noda M. Pattern of RECK CpG methylation as a potential marker for predicting breast cancer prognosis and drug-sensitivity. Oncotarget. 2016; 7:82158-69. https://doi. org/10.18632/oncotarget.8620.

60. Zhou XQ, Huang SY, Zhang DS, Zhang SZ, Li WG, Chen ZW, Wu HW. Effects of 5-aza-2'deoxycytidine on RECK gene expression and tumor invasion in salivary adenoid cystic carcinoma. Braz J Med Biol Res. 2015; 48:254-60. https://doi.org/10.1590/1414-431X20144102.

61. Cho CY, Wang JH, Chang HC, Chang CK, Hung WC. Epigenetic inactivation of the metastasis suppressor RECK enhances invasion of human colon cancer cells. J Cell Physiol. 2007; 213:65-9. https://doi.org/10.1002/jcp.21089.

62. Xue W, Kitzing T, Roessler S, Zuber J, Krasnitz A, Schultz N, Revill K, Weissmueller S, Rappaport AR, Simon J, Zhang J, Luo W, Hicks J, et al. A cluster of cooperating tumor-suppressor gene candidates in chromosomal deletions. Proc Natl Acad Sci U S A. 2012; 109:8212-7. https://doi.org/10.1073/pnas.1206062109.

63. Liu Y, Zhang X, Han C, Wan G, Huang X, Ivan C, Jiang D, Rodriguez-Aguayo C, Lopez-Berestein G, Rao PH, Maru DM, Pahl A, He X, et al. TP53 loss creates therapeutic vulnerability in colorectal cancer. Nature. 2015; 520: 697-701. https://doi.org/10.1038/nature14418.

64. Edgar RC. MUSCLE: multiple sequence alignment with high accuracy and high throughput. Nucleic Acids Res. 2004; 32:1792-7. https://doi.org/10.1093/nar/gkh340.

65. Tamura K, Stecher G, Peterson D, Filipski A, Kumar S. MEGA6: Molecular Evolutionary Genetics Analysis version 6.0. Mol Biol Evol. 2013; 30:2725-9. https://doi. org $/ 10.1093 / \mathrm{molbev} / \mathrm{mst} 197$.

66. Guindon S, Dufayard JF, Lefort V, Anisimova M, Hordijk W, Gascuel O. New algorithms and methods to estimate maximum-likelihood phylogenies: assessing the performance of PhyML 3.0. Syst Biol. 2010; 59:307-21. https://doi.org/10.1093/sysbio/syq010.

67. Hensley MR, Cui Z, Chua RFM, Simpson S, Shammas NL, Yang JY, Leung YF, Zhang G. Evolutionary and developmental analysis reveals KANK genes were co-opted for vertebrate vascular development. Sci Rep. 2016; 6:27816. https://doi.org/10.1038/srep27816. 